\title{
REFLEXIÓN SOBRE ALGUNOS PROBLEMAS DE SEMITONÍA SUBINTELECTA (MUSICA FICTA) EN LA MÚSICA DE CABEZÓN A PARTIR DE LAS FUENTES VIHUELÍSTICAS
}

\section{REFLECTION ON SOME PROBLEMS OF MUSICA FICTA IN THE MUSIC BY CABEZÓN FROM VIHUELA SOURCES}

\author{
Miguel Bernal Ripoll \\ Real Conservatorio Superior de Música de Madrid
}

\begin{abstract}
Resumen
Las antiguas fuentes españolas para tecla no siempre indican con precisión las alteraciones accidentales, bien por omisión, bien por responder a la práctica habitual de no indicarlas, dejándolas al juicio del intérprete (semitonía subintelecta). Para resolver el consiguiente problema para la práctica interpretativa, se propone examinar situaciones similares en las obras contemporáneas para vihuela, pues la tablatura de este instrumento refleja la altura precisa de las notas.

Palabras clave huela.

Semitonía subintelecta, musica ficta, Cabezón, órgano, vi-
\end{abstract}

\section{INTRODUCCIÓN}

\section{Presentación y planteamiento}

Es bien conocida la cuestión de la semitonía subintelecta en la música antigua ibérica, cuestión que se refiere a la práctica interpretativa de alterar determinadas notas a pesar de que las alteraciones no vengan expresamente escritas en la partitura, lo cual viene a ser la aplicación práctica del concepto de musica ficta recogido por las fuentes no españolas.

Esta práctica no sólo no se circunscribe a la música vocal, sino que incluso se agrava en las fuentes de música instrumental. El estudio de las fuentes impresas de música de tecla del siglo XVI y principios del XVII (es decir, Ve-

\begin{abstract}
Accidentals are not always precisely indicated in old Spanish sources for Keyboard music, either for neglect or for this corresponds to the usual practice of not indicating them, letting them to performer's opinion (semitonia subintellecta). As a way to find a solution to the resulting problem for performance practice, the purpose is to examine similar settings in contemporary works for vihuela, since tablature for this instrument actually reflects the precise pitch of the notes.
\end{abstract}

Key words huela.

Semitonia subintellecta, musica ficta, Cabezón, organ, vi-

negas, Cabezón y Correa) evidencia que es necesario añadir alteraciones no escritas. El problema es mayor en Venegas, todavía significativo en Cabezón, y menor en Correa, siendo este último más cuidadoso (aunque no totalmente exhaustivo) en señalar las alteraciones accidentales. En el siglo XVII, la carencia de impresos hace más grave el problema, toda vez que las fuentes manuscritas no autógrafas, copias personales de los organistas, tienden a simplificar todo lo que fuera obvio para ellos mismos, además de estar plagadas de errores e imperfecciones.

Si bien las reglas generales que rigen esta práctica son bien conocidas, especialmente a través de las reglas de composición de la época, su aplicación en las obras instrumentales presenta no pocas dudas, especialmente debido a la com- 
plejidad de la elaboración y diminución en dichas obras. El problema es complejo, y en mi opinión no se puede dar una solución ni sencilla, ni definitiva, ni mucho menos completa. Pero creo que sí es posible hacer algunas averiguaciones que puedan, si no aclarar totalmente algunas situaciones, al menos dar una orientación para el momento en que el intérprete deba tomar una decisión.

\section{Estado de la cuestión}

Las fuentes históricas teóricas han planteado la cuestión de la semitonía subintelecta, especialmente Tovar, Bermudo, Sancta Maria, y Cerone. Lorente y Nassarre no tratan directamente de esta cuestión, aunque de su exposición de las reglas de la composición y de cómo se deben tratar determinadas especies (intervalos) se pueden extraer conclusiones de cómo deberían corregirse determinadas situaciones, al menos a priori, sin prejuicio de que fueran válidas otras propuestas más libres respecto de las reglas generales.

La cuestión, a mi juicio, ha sido insuficientemente tratada. La sistematización y codificación de las reglas teóricas choca además con los problemas derivados de su aplicación práctica y con las dudas que plantean las fuentes musicales.

Samuel Rubio ${ }^{1}$ hace una exposición bastante sistemática, completa y ordenada de las reglas dadas por Tovar, Bermudo y Sancta Maria, pensando en un ámbito de aplicación vocal. Charles Jacobs y James Wyly recogen las reglas dadas por Bermudo y Sancta Maria reivindicándolas para la música de tecla. Jacobs $^{2}$ las cita muy brevemente, mientras Wyly ${ }^{3}$ las recoge de manera más extensa, haciendo referencia a los trabajos de $\mathrm{Ru}$ bio y Jacobs. Sólo mucho más tarde Mariano Lambea ${ }^{4}$ recoge las reglas dadas por Cerone, también en un ámbito vocal.

Kastner reflexiona sobre algunos problemas de semitonía, comparando fuentes de tecla, y aplicando consideraciones organológicas (existencia o no de algunos semitonos en los teclados, temperamentos). Rechaza la posibilidad de establecer unas conclusiones dogmáticas y codificadas, como las que había expuesto W. Apel anteriormente, apelando al oído y al bon sens para solucionar los problemas.

El Diccionario de la Música Española e Hispanoamericana no dice nada de la cuestión. El New Grove dedica un artículo de extensión media, que no cita las fuentes históricas teóricas españolas, ni los problemas que presentan las fuentes musicales.

1 RUBIO, 1956: 54-86.

2 JACOBS, 1968: 71-81.

3 WYLY, 1964: 247-271.

4 LAMBEA, 1996.
Silbiger ${ }^{5}$ solo hace una referencia general al problema en la música de tecla, aunque de forma práctica y abogando también por soluciones intuitivas, señalando que "una solución más tonal es sin duda más cercana a la correcta". El capítulo del libro (escrito por Robert Parkins) dedicado a la música española y portuguesa no entra en el tema. Aunque el trabajo de Iina-Karita Hakalahti ${ }^{6}$ está aplicado a Correa, su introducción al tema es una excelente aproximación, con un resumen de las reglas dadas por Bermudo y Sancta María, así como un estado de la cuestión.

Haciendo referencia al problema en la música vocal, encontramos sólo un autor que cita, aunque muy brevemente, algunas tablaturas instrumentales. Se trata de Vincent Arlettaz ${ }^{7}$, aunque de las españolas solo cita de pasada a $\mathrm{Pi}$ sador, en el que cree encontrar algunas cadencias que llama "modales" porque parece no aplicar las reglas de semitonía en las clausulas que deben ser sostenidas, para señalar que las demás tablaturas españolas de vihuela no tienen tales cadencias "modales". Esto lo explica Arlettatz señalando que el propio Pisador era considerado poco "profesional" por sus contemporáneos, y su obra sería un ejemplo de los "barbarismos" que Bermudo había señalado en algunas tablaturas de vihuela.

Junto a los trabajos literarios, habría que incluir a las ediciones de música de tecla, que aportan las sugerencias prácticas para los organistas. Pero normalmente los editores no se extienden en explicar el porqué de sus sugerencias (una edición práctica quizás tampoco es el lugar para ello), siempre criticadas porque no hay dos intérpretes o musicólogos que estén de acuerdo en este problema.

\section{Planteamiento y metodología}

Este trabajo se circunscribirá a la música instrumental del siglo XVI, especialmente a la de Antonio de Cabezón. A este respecto, la propuesta de este artículo es examinar las obras de los vihuelistas, pues en la cifra para vihuela se representan exactamente las alturas, no habiendo por tanto $a$ priori problema para el intérprete en la semitonía. Se trabaja con la hipótesis de que la comparación de construcciones similares en la literatura de tecla y las de vihuela podrá aportar alguna luz a esta cuestión.

Se advierte que necesariamente el trabajo no es exhaustivo, sino tan sólo una aproximación al problema que permita al menos al intérprete una herramienta para tomar

5 SILBIGER, 2004: 8-9.

6 HAKALAHTI, 2008: 264-280.

7 ARLETTATZ, 2000. 
decisiones en uno de los aspectos más difíciles de la práctica interpretativa de la música antigua ibérica. De hecho, la magnitud de la casuística solo permite plantear algunos casos concretos.

El procedimiento será presentar situaciones en obras de Cabezón que nos plantean dudas, para buscar a continuación pasajes similares en vihuelistas para ver cómo los resuelven, suponiendo que en las fuentes vihuelísticas sabemos exactamente cuál es la solución, pues la tablatura para vihuela nos devuelve las alturas precisas.

Es necesario tener en cuenta que en el siglo XVI la actual notación silábica tenía solo un valor relativo. La notación absoluta para las notas musicales (llamados "signos") combinaba la alfabética y la silábica, empleando la letra correspondiente seguida de las posibilidades de solmisación. En este trabajo se emplea la notación actual (que se pondrá siempre en mayúscula) para las notas musicales, pero añadiremos entre paréntesis la notación antigua.

\section{Tabla 1: Correspondencia entre la notación antigua y la moderna}

$\begin{array}{cl}\text { Notación moderna } & \text { Notación antigua } \\ \mathrm{Do} & \text { Cesolfaut } \\ \mathrm{Re} & \text { Delasolre } \\ \mathrm{Mi} & \text { Elami } \\ \mathrm{Fa} & \text { Fefaut } \\ \mathrm{Sol} & \text { Gesolreut } \\ \mathrm{La} & \text { Alamire } \\ \mathrm{Si} & \text { Befabemi }\end{array}$

Las voces Fa y Mi denotaban las dos posibilidades de una misma nota, un semitono más bajo o más alto. Así, el "mi de fefaút" denotaría el Fa\#, el "fa de Elami" el Mib, etc.

En las transcripciones se siguen las convenciones actuales, de las que destacamos el empleo moderno del signo de becuadro en lugar de bemol y sostenido cuando se trata de hacer natural una nota alterada, cuando la convención antigua es emplear siempre sostenido para elevar un semitono y bemol para bajar. Según esa convención, para hacer el Si natural en una obra con un bemol en la armadura, se emplea el sostenido, cuando actualmente utilizamos el becuadro (en origen ambos signos eran el mismo). Como se ha dicho, empleamos la convención moderna, mediante le becuadro.

\section{Crítica de fuentes}

Las fuentes primarias del estudio serán por tanto los libros españoles de vihuela impresos en el siglo XVI (Mu- darra, Fuenllana, Pisador, Valdarrábano, Milán, Daza) junto con el manuscrito Ramillete de flores, y las obras de Cabezón editadas por Venegas y Hernando.

La investigación debe comenzar por una crítica de la validez de las fuentes empleadas, a saber, la literatura vihuelística. La suposición de que la resolución de los problemas de semitonía por los vihuelistas sería un modelo a considerar para la resolución de los problemas similares que se encuentran en las fuentes de tecla debe ser matizada con las siguientes consideraciones.

En primer lugar, en dichas fuentes, al entablar la polifonía, los autores podrían haberla entablado tal cual estaba en la fuente vocal, es decir "tal como se escribe", y corregir luego el intérprete - a pesar de lo escrito- según la práctica habitual. Esta práctica podría afectar no solo a las transcripciones vocales sino incluso a las fantasías.

Por otro lado, podrían representar una práctica particular de los vihuelistas, que quizás no siempre trataran la semitonía de manera idéntica en otros medios instrumentales. La conocida "Fantasía que contrahace la harpa a la manera de Ludovico" de Mudarra podría ser un ejemplo de la práctica de una semitonía propia de un intérprete particular en un instrumento determinado. Esto apoyaría la idea de que diferentes medios instrumentales pueden tener diferentes soluciones para la semitonía.

No puede descartarse también que pudiera haber errores en las fuentes vihuelísticas, bien procedentes del proceso de edición e impresión, bien de los propios autores. Se recuerda la observación referente a Pisador hecha más arriba citando a Arlettaz.

En cualquier caso, se puede aceptar el punto de partida de que la semitonía está razonablemente bien resuelta en las fuentes vihuelísticas y que la práctica tendría validez general para la música de órgano, a condición de no caer en la tentación de querer establecer normas dogmáticas, pues la experiencia parece demostrar que tal simplificación no es posible.

\section{EL SI BEMOL (BEFABEMI BEMOL)}

Como se ha dicho al exponer las intenciones de este trabajo, no estoy en condiciones de dar una exposición sistemática de todos los problemas de semitonía en las ediciones originales cabezonianas. La magnitud de la casuística es tal, que es necesario mucho más tiempo y espacio para abarcarlo. En este trabajo hay lugar tan solo para exponer la propuesta metodológica en algunos casos concretos.

Algunos de los problemas más difíciles siempre se me presentan con nuestra nota "SI" (Befabemi), que es el $6^{\circ}$ grado en el primer tono o el $4^{\circ}$ grado en sexto tono. Tenemos 
tendencia natural a bemolizarla, debido a nuestro oído tonal. En la reflexión sobre esta cuestión, encontramos por un lado las observaciones de Bermudo y de Correa advirtiendo contra el uso excesivo del bemol:

“...Pues si no fvere por necesidad: no se ha de hacer el dicho bmol [en los modos $5^{\circ}$ y $\left.6^{\circ}\right]^{\prime \prime} 8$

"Quieren algunos estos dos modos [5 $5^{\circ}$ y $\left.6^{\circ}\right]$ cantarlos, y tañerlos siẽpre por bemol: con lo qual qebrantan el diapaßõ de ambos" ".

"...el be quadrado en estos dos tonos, es vn despertador y vna espuela que aviva y despierta a los que cantan; $y$ assi veran que quando canta el choro el fabordon del sexto tono; si se le tañe por bemol al boluer a entrar en el la de alamire, entran tan blanda y floxamente que casi lo hazẽ fa: semitono de fefaut; $y$ assi tañendo por be quadrado el dicho sexto [f. 3 v.] tono, paresce que abiuã y despiertan haziendo fuerte el la de alamire y formando la dicha tercera mayor en su legitima cãtidad, como se deue formar: y en estas ocasiones es bueno vsar del sexto tono por be quadrado" 10
Por otro lado, están las reglas que prescriben el uso del bemol para evitar el tritono, que evidencian que nuestro oído "tonal" - como quiere Silbiger, vide sopra - no está tan lejano de la práctica de la época. No en vano la "tonalidad" se deriva de la práctica de determinadas pautas que por uso acabaron estableciéndose como norma ${ }^{11}$.

En la música de corte más eclesiástico, y por tanto más tradicional, sería todavía más corriente no "bemolizar". Recordemos cómo Sancta María refiere como una excepción el hecho de que en Toledo se cantara el saeculorum del $6^{\circ}$ tono por bemol:

"Asi mismo el seculorum del quinto tono, es contrario en la propriedad al [f. 65] quinto tono, que Se canta y tañe conforme a su propriedad y naturaleza, en el que se canta y tañe conforme al saeculorum, se canta y tañe por bequadrado, y el que se canta y tañe conforme a su propriedad y naturaleza, se canta y tañe por Bemol, aunque en el Arçobispado de toledo, tambien el seculorum se canta por Bemol. ${ }^{12}$ ",

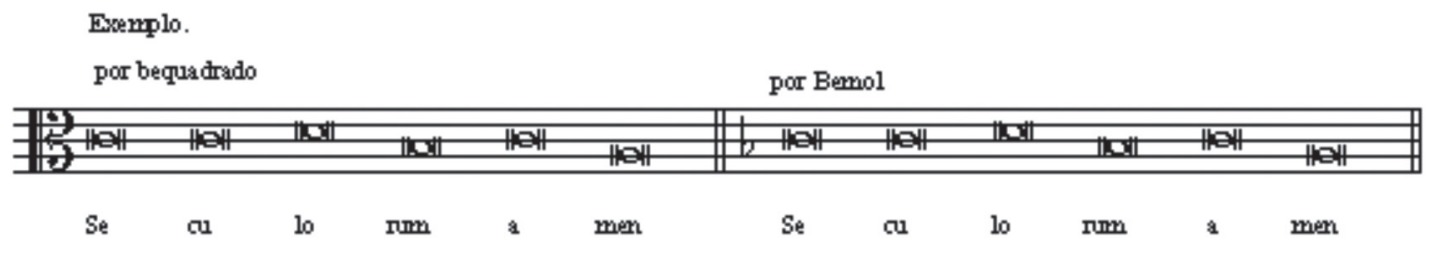

Ilustración 1: Ejemplo del seculorum del $6^{\circ}$ tono por natura y por bemol según Sancta María

En realidad, podríamos considerar que se trata de dos modos distintos, a saber lydio y jonio.

También es de señalar que con la glosa se producen situaciones que crean duda, y sin duda serían dudosas también para los propios músicos de la época.

\section{El $6^{\circ}$ grado en el primer tono en escala descendente tras la nota final}

Cuando en los tonos primero o segundo, cuando después de la nota final de un periodo o de la propia obra hay

8 BERMUDO, 1555: 39v.

9 Ibíd:: $72 \mathrm{v}$.

10 CORREA, 1626: 3r-3v. una escala descendente, se suscita la duda de si el sexto grado debe estar alterado descendentemente, es decir, si debe ser Si bemol (Befabemi bemol). En términos del sistema hexacordal, diríamos que el problema reside en si convertimos o no esa nota en un fa, empleando por tanto la propiedad de bemol.

Se trata de situaciones como las siguientes (marcadas con *):

11 Advierto, en cualquier caso, que en mi opinión no se puede decir que la tonalidad esté ya ahí, hay indicios y situaciones que más tarde se convertirán en la tonalidad, pero que en la época no se sabía todavía a dónde iban a llevar.

12 SANCTA MARIA, 1565, I: 65. 


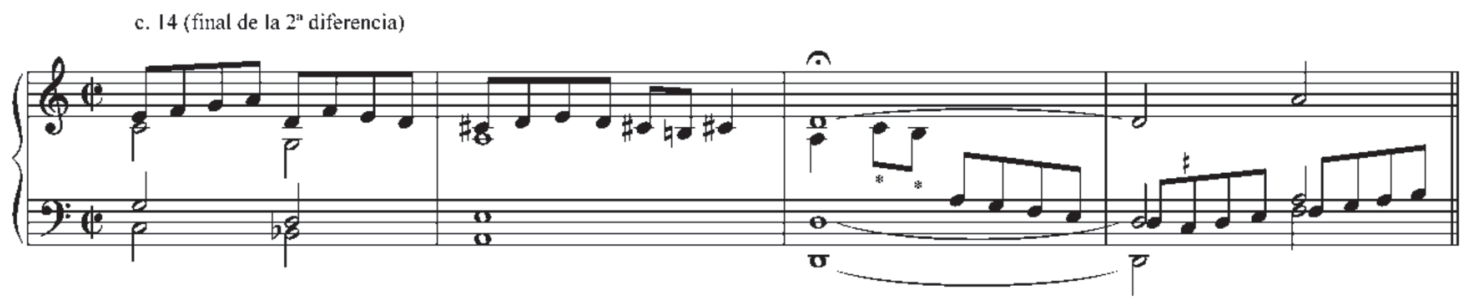

Ilustración 2: Cabezón, Diferencias sobre La Pavana Italiana ${ }^{13}$

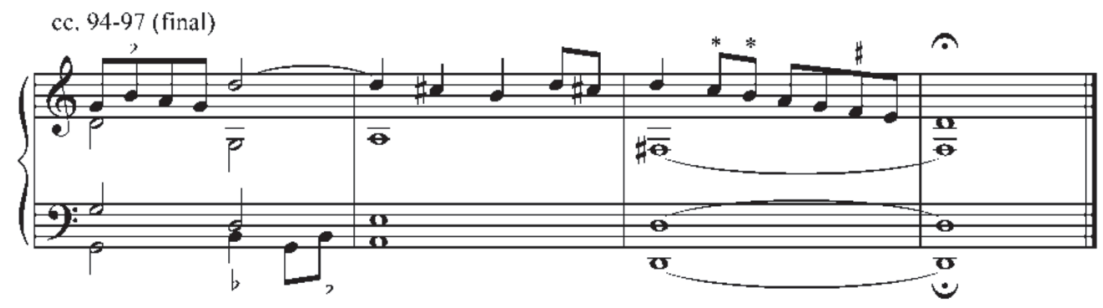

Ilustración 3: Cabezón, Diferencias sobre Madama me demanda ${ }^{14}$.

Nuestro oído tonal tenderá a hacer bemol el $6^{\circ}$ grado (como en la escala menor melódica), o a lo sumo hacer de nuevo sostenido el $7^{\circ}$ grado. Por otro lado, nuestra necesidad de que suene "extraño", o diferente a lo tonal nos sugiere hacerlo sin alterar, pero podría tratarse de una mera construcción nuestra, por un prejuicio de que toda solución que se parezca a lo tonal debe descartarse, cuando en definitiva lo "tonal” se derivó de estas prácticas.
Sin embargo, si observamos situaciones similares en los vihuelistas, encontramos que en dicha escala descendente después de la nota final nunca se alteran ambos grados $6^{\circ}$ y $7^{\circ}$, resultando la escala natural del tono $1^{\circ}$ ó $2^{\circ}$. Esto se corrobora en los siguientes ejemplos. 


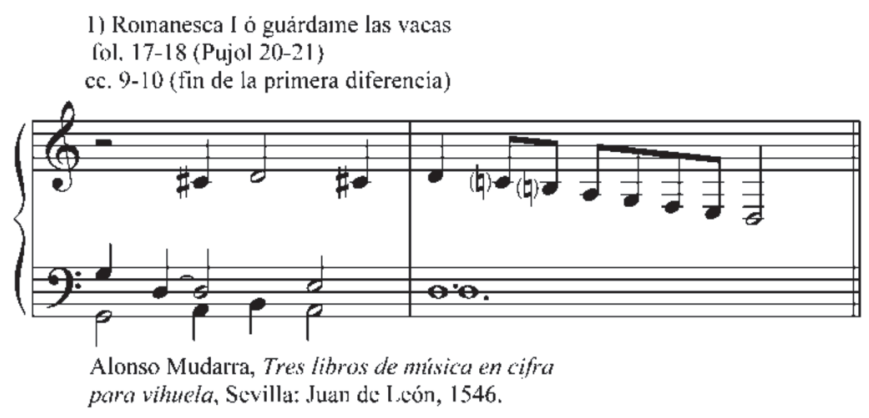

Ilustración 4: Mudarra, Romanesca I o Guárdame las Vacas ${ }^{15}$

cc. 33-35, tinal de la tercera diferencia (c. 23-24 en Rey)

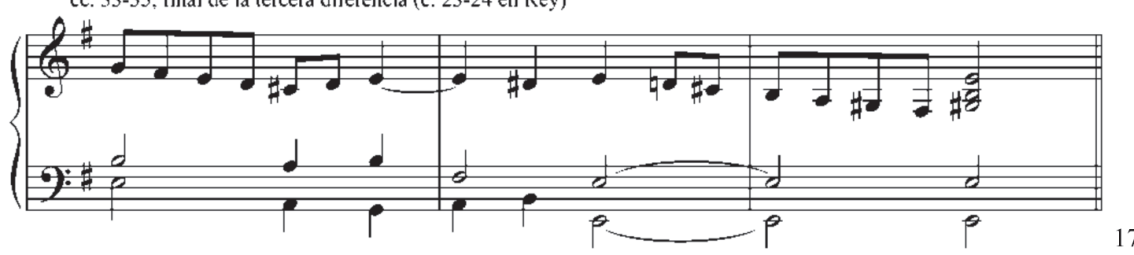

Ilustración 5: Francisco Páez. Seis diferencias de Bacas $^{16}$ (primer tono, final en Elami)

17

Los ejemplos anteriores corresponden a obras de la tipología de las diferencias, donde frecuentemente se dan frases y periodos cerrados. Pero también encontramos esta pauta en obras de otras tipologías, como en el villancico siguiente:

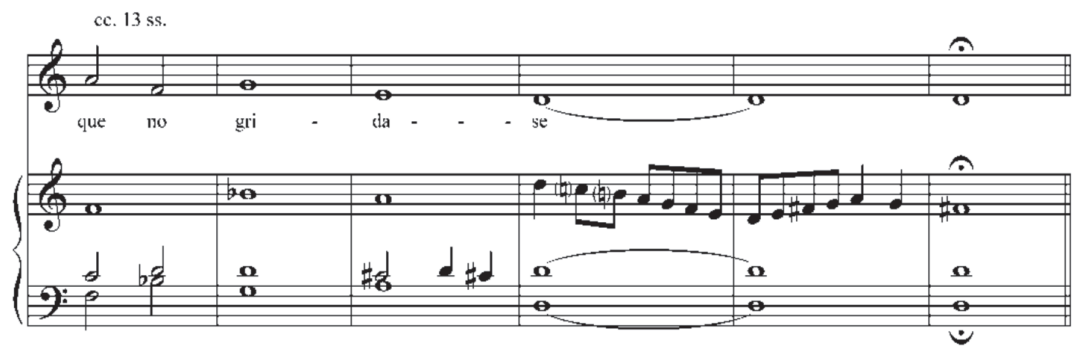

Ilustración 6: Mudarra, Villancico $\mathrm{V}^{18}$

15 MUDARRA, 1547: 17-18; 1984: 20-21

16 Ramillete de flores: $273 \mathrm{v}-275 ; 1982: 89-92$.

17 N.B el barrado original de esta pieza tiene valor meramente métrico, realmente el ritmo real corresponde a compases de proporción de tres mínimas al compás, aunque se ha escrito en "compases" cuyo valor es un semibreve, de manera que el espacio entre barras sería dos tercios de un compas. Esta práctica es habitual en otros escritos de vihuela (véase la pavana de Pisador, fol. V, por ejemplo). La transcripción de Rey refleja el ritmo "real".

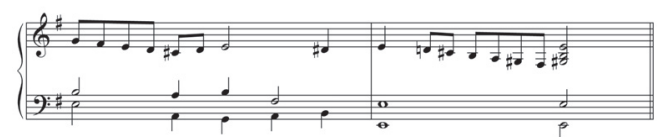

18 MUDARRA, 1546: 55-55v (=108-108v); 1984: 131. 
No sólo encontramos esta situación en final de obra, ocurre también en final de frase:

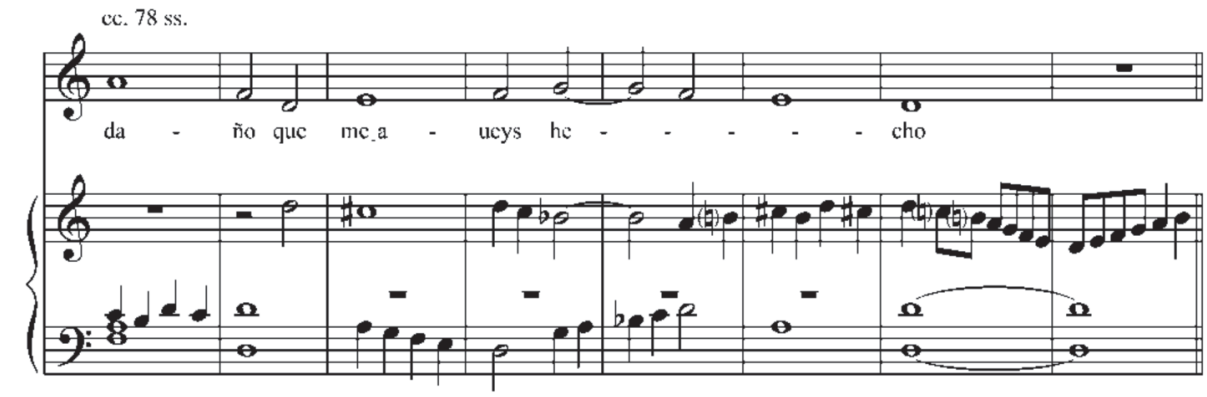

Ilustración 7: Mudarra, Villancico III ${ }^{19}$

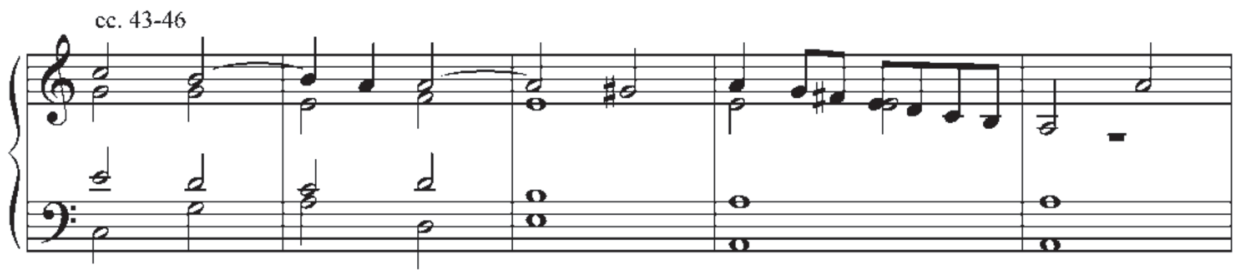

Ilustración 8: Daza, Fantasía 2, segundo tono final en La (Alamire) ${ }^{20}$

Otras veces ocurre en el bajo, siempre con el mismo resultado de estar sin alterar:

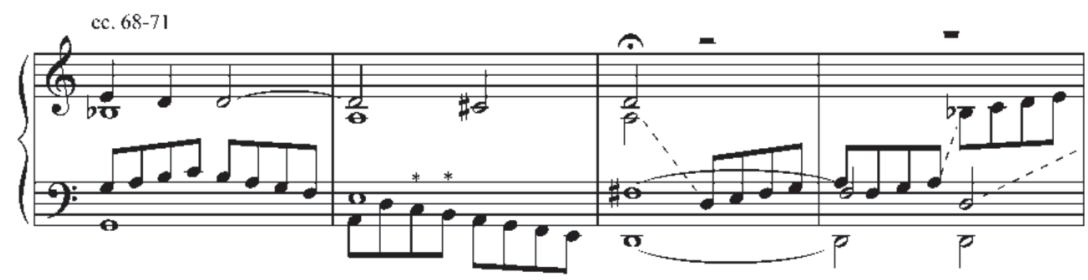

Ilustración 9: Cabezón, diferencias sobre Las Vacas ${ }^{21}$

Se han consultado minuciosamente las obras de Valderrábano, Pisador, Mudarra, Ramillete, Milán y Daza. En Pisador, Milán y Daza no se da esta situación, y en los otros autores no se ha encontrado caso alguno en que se altere el $6^{\circ}$ grado descendentemente en estas escalas tras la nota final. Sin embargo, cuando no es en una nota final, sí encontramos alterado descendentemente el sexto grado en la escala descendente: 


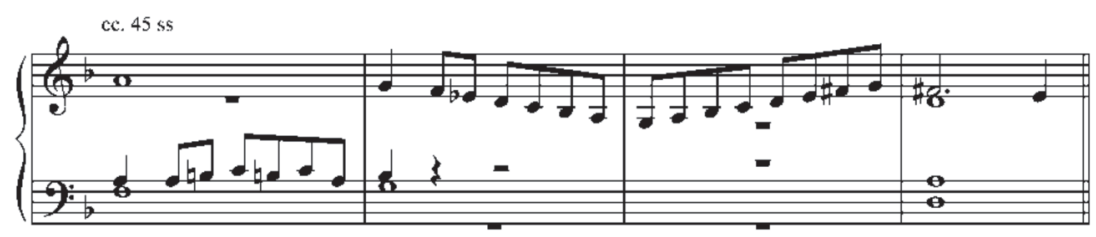

Ilustración 10: Mudarra, Pavana ${ }^{22}$

Lo mismo ocurre en el caso siguiente, aunque es necesario advertir que ocurre dentro de una misma consonancia, y que estamos en séptimo tono: en la parte vocal, en canto de órgano, final en La (Alamire), sin alteraciones en la clave, en cuyo caso el grado que se altera es el segundo de dicho tono.

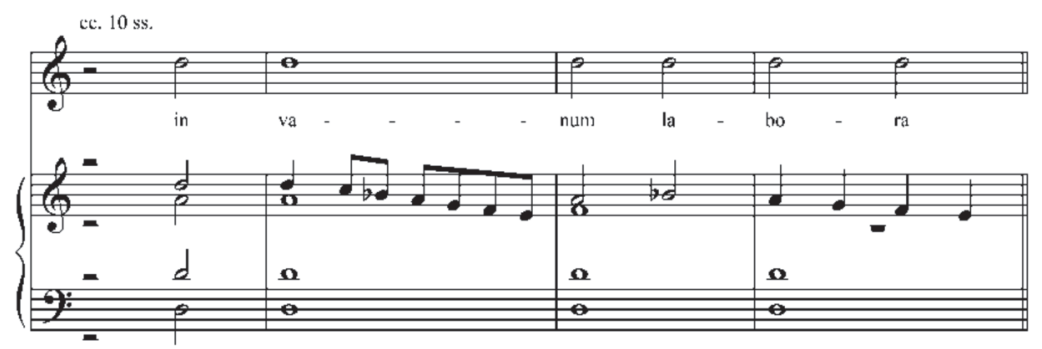

Ilustración 11: Mudarra, Psalmo I por el séptimo tono ${ }^{23}$

Por el resultado anterior, en mi opinión sí deberíamos hacer bemol en el lugar marcado * del ejemplo siguiente:
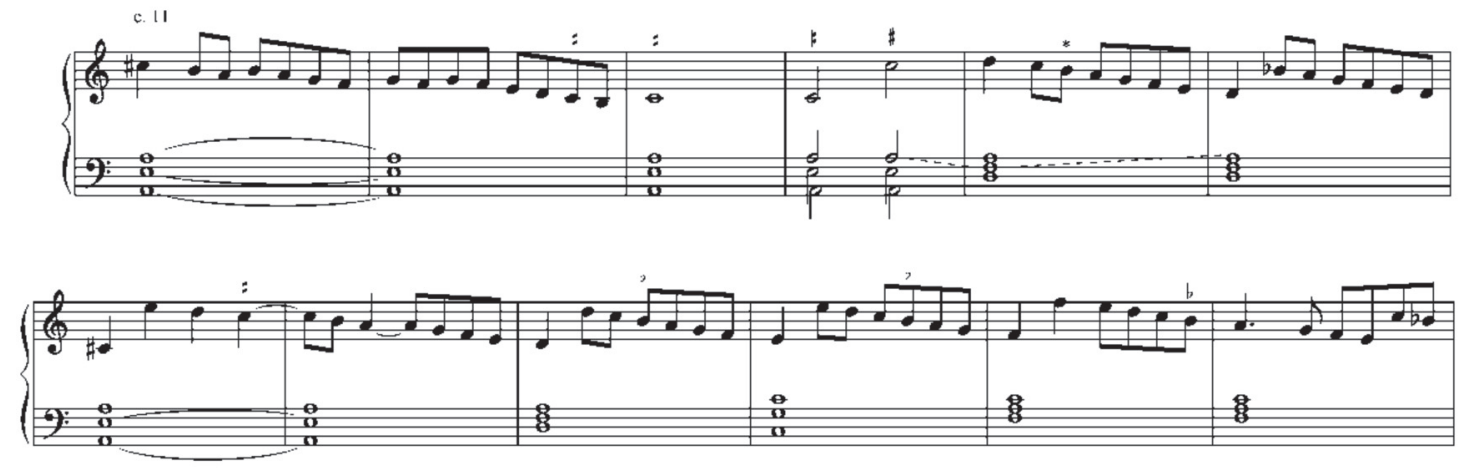

Ilustración 12: Cabezón, Romance Para quién crié yo cabellos ${ }^{24}$ 
A la vista de los resultados anteriores, se puede concluir que ambos grados VI y VII, en la escala descendente del tono después de una nota final, deben estar sin alterar. sin embargo, cuando la escala descendente no es después de una nota final se debe alterar descendentemente el sexto grado

\section{$6^{\circ}$ GRADO BEQUADRO EN LA CONSTRUCCIÓN IV-V-I}

Otro problema que se presenta con el sexto grado en los tonos $1^{\circ}$ y $2^{\circ}$ se presenta en la siguiente sucesión de consonancias.
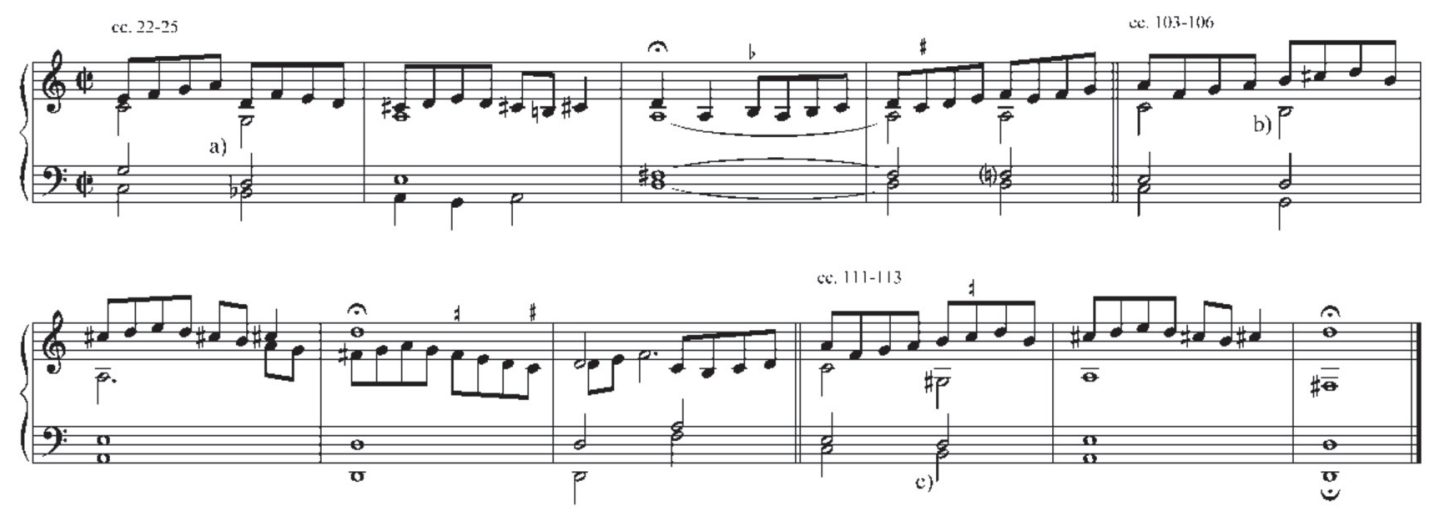

Ilustración 13: Cabezón, Diferencias sobre la Pavana Italiana ${ }^{25}$

El Si (Befabemi) ¿debe ser bemol (Bb)? Normalmente así debería ser, para que se cumpla la regla de que un intervalo imperfecto antes de otro perfecto debe estar lo más cerca posible de este, la llamada "ley de la máxima proximidad" que recogen las fuentes historiográficas en un intento de sistematizar las normas de la época ${ }^{26}$. De manera particular, la décima Sol-Si (G-Bb) que se dirige a la octava La-La (A-A) debe ser menor, o la sexta Si-Sol (B-G) debe ser mayor, en ambos casos para estar más cerca de la octava La-La (A-A).

Así ocurre en a) del anterior ejemplo. En los casos a) y b) dicha nota no está alterada, en el primer caso porque melódicamente va seguido de Do\# (Cesolfaut sostenido C\#), no pudiéndose cumplir la norma antes expuesta. En el segundo porque el Sol es sostenido (Gesolreut sostenido, G\#), satisfaciéndose así la regla de que la sexta antes de la octava sea mayor.
Una aclaración: se puede pensar que dicha construcción se parece a la actual cadencia perfecta IV-V-I. Pero es exactamente lo contrario: nuestra cadencia perfecta se parece a esa construcción, que a fuerza de ser empleada se convirtió en una pauta habitual. Ahora es fácil ver que esa construcción dio lugar a la cadencia perfecta, pero querer ver ya una intención tonal funcional ahí es - como diría Bergson - un "espejismo del presente en el pasado" 27.

Bajo estas consideraciones, en el caso siguiente y otros similares se podría pensar en hacer el si b $(\mathrm{Bb})$.
25 CABEZ̄ón, 1578: 190v-192; 2010: 45-49. 26 RUBIO, 1956: 78.
27 Esta es una reflexión que ya he hecho en un trabajo anterior, cfr. Bernal, 2005. 

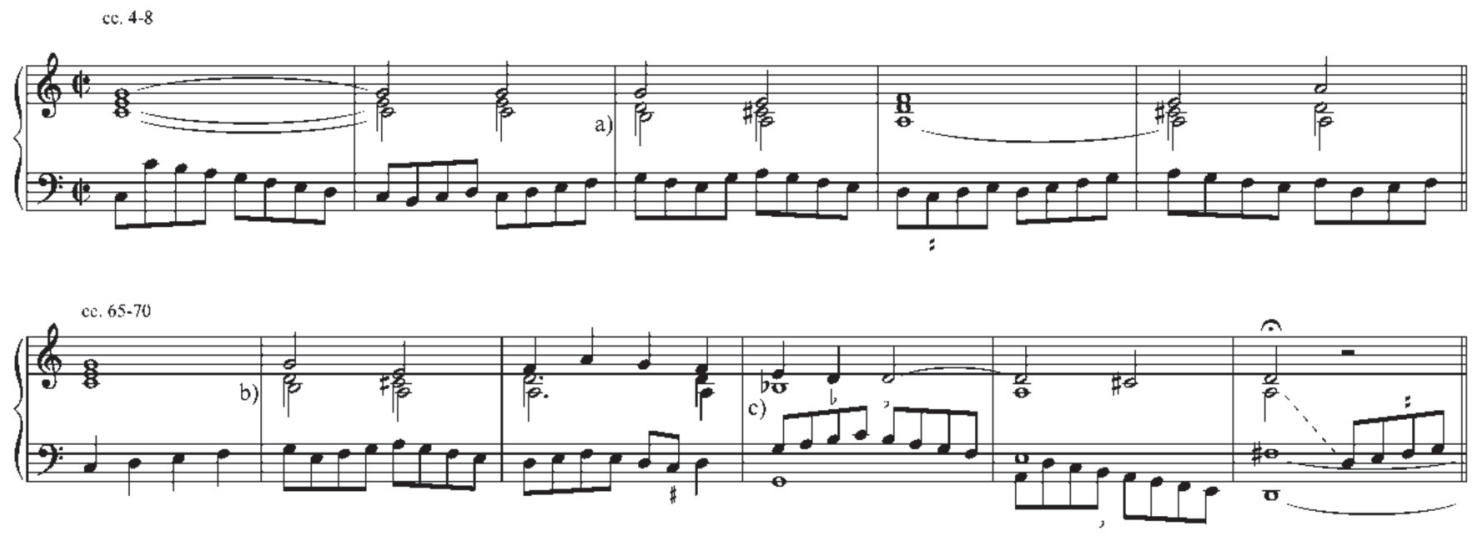

Ilustración 14: Cabezón, diferencias sobre las Vacas ${ }^{28}$

En a) y b) del anterior ejemplo tenemos un si natural, en el que podríamos suponer se ha obviado el bemol que sí haríamos en la práctica interpretativa. En realidad no se trata de un caso de "IV-V-I", en a) no hay un reposo en el I (final del tono), sino en el $\mathrm{V}$ grado del tono (es la mediación de la frase completa). Tampoco estamos en un reposo en el I en b), la cadencia final IV-V-I está en c), donde sí hay expresamente indicado un bemol.

En los anteriores casos a) y b) podemos justificar la ausencia del bemol por un virtual movimiento si-do\#, suponiendo cruce de voces.
En cualquier caso, se encuentran en la literatura para vihuela algunos casos en los que no se altera con bemol el si (Befabemi) en situaciones similares. ¿Error u omisión, o prueba de una práctica menos estricta? En el caso b) del ejemplo siguiente, el si natural está justificado por estar sin embargo el sol\# (Gesolreut sostenido $\mathrm{G \# ).} \mathrm{El} \mathrm{caso} \mathrm{a)} \mathrm{es} \mathrm{am-}$ biguo al estar a tres voces, pero en c) no se cumple la regla de que la sexta antes de la octava deba ser mayor.

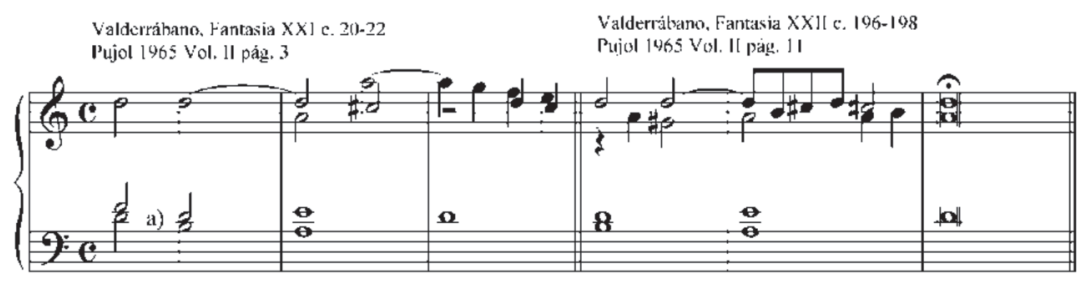

b)

Valderrálbant. Hamtasia $X X[1]$ c. $71-74$ Pujol 1465 Vol. 11 prage. 1.3

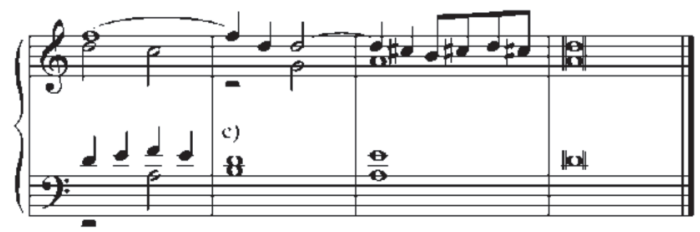

Ilustración 15: Ejemplos de obras de Valderrábano 


\section{EL VII GRADO Y EL GRADO INFERIOR A LA CLÁUSULA SOSTENIDOS}

\section{Escala ascendente antes de cláusula sostenida}

Se examina ahora el caso de una cláusula sostenida precedida por una escala ascendente. Veamos qué le sucede a la nota inferior a la final.
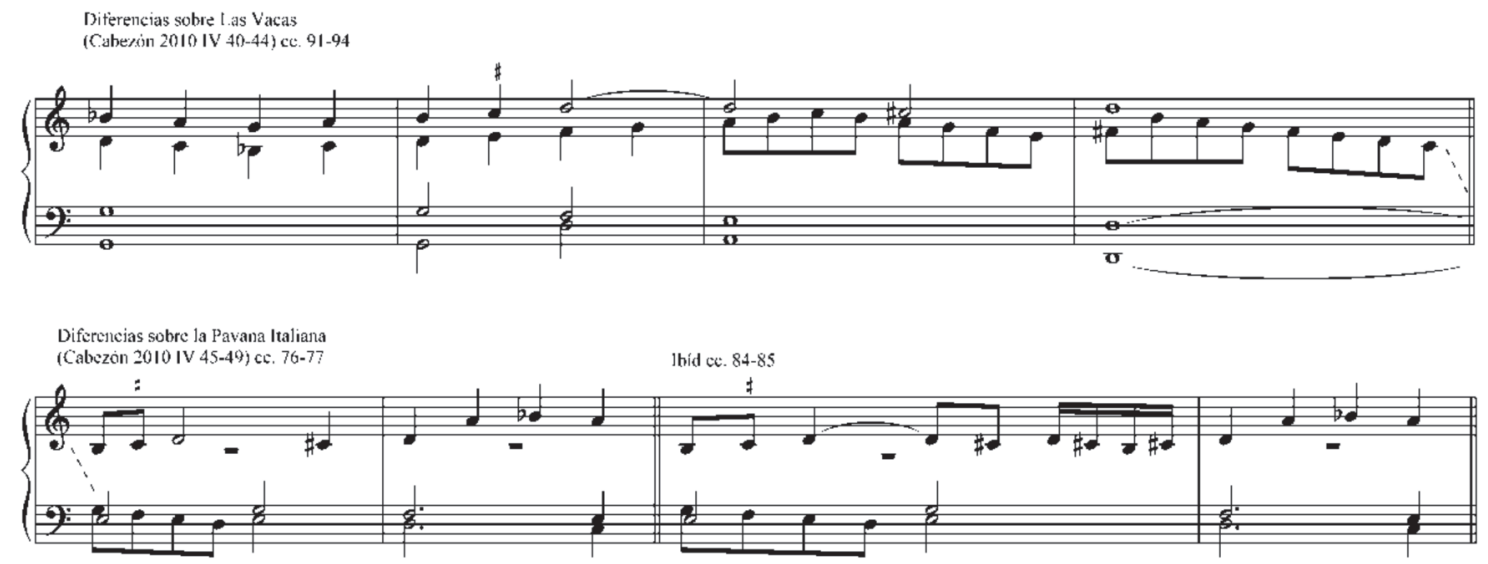

Ilustración 16: Ejemplos de VII grado ascendiendo a I en Cabezón

Si se tiene en consideración las voces de tiple y alto del primer ejemplo o las de tiple y bajo de los dos siguientes, se comprueba corresponde a un tipo de cláusula descrito por Sancta Maria a dos voces ${ }^{29}$, al que se ha añadido el VII grado como nota de paso rellenando la tercera:

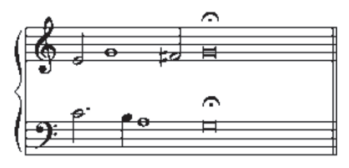

Ilustración 17: Cláusula descrita por Sancta Maria

La tercera antes de la cláusula está glosada, en ocasiones viniendo de un movimiento ascendente más extenso por grados conjuntos. Examinando la obra de los vihuelistas se comprueba que el punto anterior a la cláusula es también sostenido, lo que justifica la sugerencia de semitonía

29 SANCTA MARIA, 1565, I: 67. que se ha hecho en los ejemplos anteriores de Cabezón. Si bien esto es lo que ocurre en la mayoría de los casos, para ser rigurosos hay que admitir que se encuentran en Milán algunos ejemplos de no alterar dicha nota. A continuación, ejemplos de Valderrábano y Daza que alteran ascendentemente, y un ejemplo de Milán donde no se altera ${ }^{30}$ (Nota Bene: el ejemplo de Daza se ha transportado un tono más bajo)

30 Se encuentran situaciones similares en Valderrábano: Fantasia XXV 40-43 (VALDERRÁBANO, 1965, II: 16-17), Fantasia XXVII cc. 93-95 (Ibíd: 21-24), Canción IV cc. 35-38, 46-48 (Ibíd.: 38-39), soneto XIV cc. 6-8 (Ibíd.: 51-52, Soneto XXII cc. 5-8 (Ibíd.: 59), Pavana cc. 158-160 (Ibíd:: 65-68). Del mismo modo en Milán: Fantasia II cc. 16-18 (MILÁN, 1974: 3-4), Fantasia VI (Ibíd.: 9-12), Fantasia XXIX cc. 101-103 (Ibíd:: 97-99), así como otros ejemplos de no alterar dicha nota en Pavana I c. 21-23 (Ibíd.: 63), Fantasia XXIX cc. 62-62 (Ibíd:: 97-99) aunque en esta última obra sí se altera en una situación similar pocos compases después. 

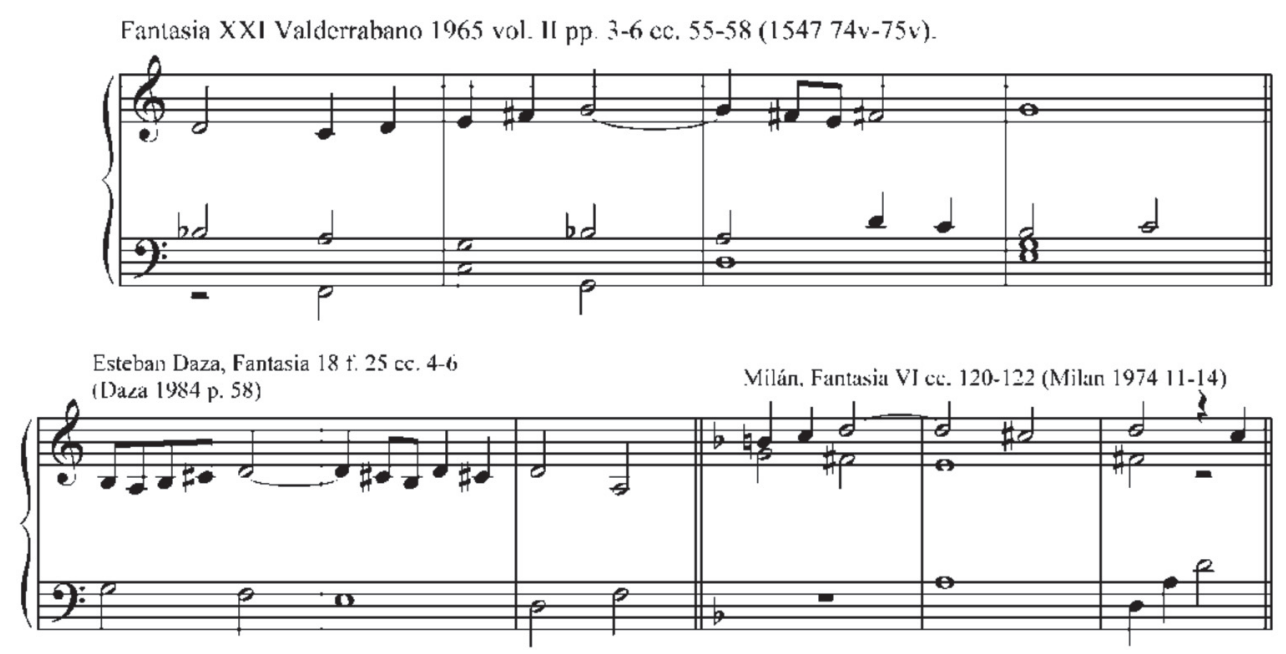

Ilustración 18: ejemplos de alteración ascendente del VII grado cuando va al I

\section{BORDADURA EN RE, SOL O LA.}

El caso de una bordadura descendente, es decir, una melodía que nota que se separa y vuelve a la nota, en parte débil, con un movimiento de un grado ${ }^{31}$. Cuando tienen lu- gar en las notas re, sol y la del hexacordo (no confundir con las notas absolutas), parece lógico que el movimiento sea de semitono. A continuación se aportan algunos de los numerosísimos ejemplos ${ }^{32}$ en Cabezón.
31 Lorente lo denomina "paso de vuelta", y los considera malos en el contrapunto (LORENTE, 1672: 311).
32 Más ejemplos de situaciones similares en: Diferencias sobre Las Vacas (CABEZÓN, 2010, IV: 40-44) cc. 7, 15, 47, 67. Diferencias sobre la Pavana Italiana (Ibíd.: 45-49) cc. 13, 17, 21, 25, 40, 105. Diferencias sobre La Gallarda Milanesa (Ibíd.: 50-52) cc. 33, 42, 46, 52, 54, 62, 66. Discante sobre La Pavana Italiana (Ibíd.: 58-61) cc. 16, 36, 50, 63-64. Diferencias sobre La dama le demanda (Ibíd.: 62-65) cc. $28,49,81$. 


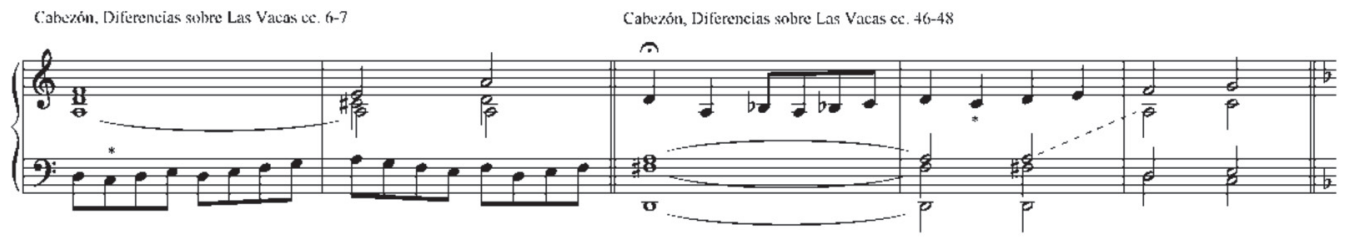

Cabezon, Diferencias sobre la (iallarda Milanesa cc, $41-43$
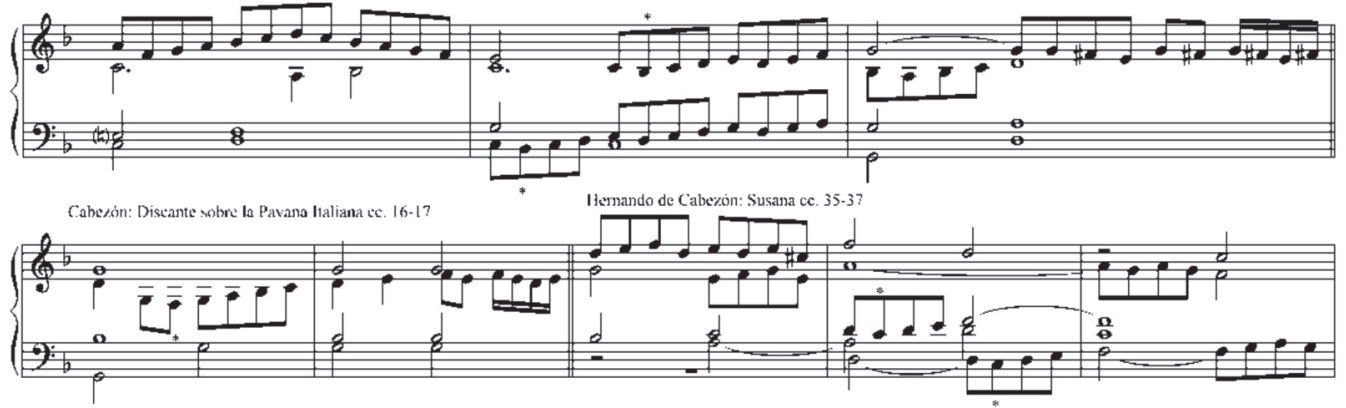

Ilustración 19: ejemplos de bordaduras o "pasos de vuelta" en Cabezón ${ }^{33}$

Se han marcado con $*$ las bordaduras donde parece lógico que el movimiento sea de semitono. Siguiendo la propuesta metodológica de este trabajo, se ha examinado todas las bordaduras en re/sol/la en contenidas en un grupo de obras de Valderrábano, excluyendo las que darían lugar a octava falsa. La tabla siguiente indica cuándo ese movimiento es de semitono (para lo cual se emplea el sostenido), en qué valores se mueve la glosa, a qué voz del hexacordo corresponde, y el intervalo que forma con otras voces, principalmente con el bajo.

Tabla 2: estudio de las bordaduras en Valderrába

\begin{tabular}{|l|l|l|l|l|}
\hline Lugar & Semitono & Valores de la glosa & Voz & Intervalo \\
\hline Fantasía XXI c. 13 & Sí & Semínima & Re & $8^{\text {a }}$ \\
\hline Fantasía XXI c. 90 & Sí & Corchea & Sol & Unísono \\
\hline Fantasía XXII c. 4 & Sí & Semínima & La & $5^{\text {a }}$ \\
\hline Fantasía XXII c. 7 & Sí & Corchea & Re & $8^{\text {a }}$ \\
\hline Fantasía XXII c. 71 & Sí & Semínima & La & $5^{\text {a }}$ \\
\hline Fantasía XXII c. 80 & Sí & Corchea & Re & $8^{\text {a }}$ \\
\hline Fantasía XXII c. 119 & Sí & Semínima & La & Unísono \\
\hline Fantasía XXII c. 131 & Sí & Semínima & La & Unísono \\
\hline Fantasía XXIII c. 32 & Sí & Semínima & Sol & Unísono \\
\hline Fantasía XXIII c. 42 & Sí & Corchea & La & $8^{\text {a }}$ \\
\hline Fantasía XXIII c. 58 & Sí & Corchea & Sol & $5^{\text {a }}$ \\
\hline Fantasía XXIV c. 77 & Sí & Semínima & Sol & Unísono \\
\hline Fantasía XXIV c. 85 & Sí & Semínima & Sol & $8^{\text {a }}$ \\
\hline Fantasía XXVIII c. 11 & Sí & Corchea & Sol & $5^{\text {a }}$ \\
\hline
\end{tabular}

33 CABEZÓN, 2010, IV: 40-44, 45-49, 50-52, 23-28. 


\begin{tabular}{|c|c|c|c|c|}
\hline Fantasía XXVIII c. 54 & Sí & Corchea & $\mathrm{La}$ & $8^{\mathrm{a}}$ \\
\hline Fantasía XXIX c. 61 & Sí & Semínima & Sol & $8^{\mathrm{a}}$ \\
\hline Fantasía XXIX c. 62 & Sí & Corchea & Sol & $8^{\mathrm{a}}$ \\
\hline Canción IV c. 2 & Sí & Semínima & $\mathrm{La}$ & Unísono \\
\hline Soneto XXIII c. 2 & Sí & Semínima & $\operatorname{Re}$ & $8^{\mathrm{a}}$ \\
\hline Soneto XXVI c. 29 & Sí & Semínima & $\operatorname{Re}$ & $8^{\mathrm{a}}$ \\
\hline Soneto XXVI c. 38 & Sí & Semínima & $\operatorname{Re}$ & $8^{\mathrm{a}}$ \\
\hline Soneto XXVI c. 48 & Sí & Semínima & $\operatorname{Re}$ & $8^{\mathrm{a}}$ \\
\hline Pavana c. 88 & Sí & Semínima & $\mathrm{La}$ & $8^{\mathrm{a}}$ \\
\hline Pavana c. 115 & Sí & Semínima & $\operatorname{Re}$ & $8^{\mathrm{a}}$ \\
\hline Diferencias sobre la Pavana c. 95 & Sí & Corchea & $\operatorname{Re}$ & $5^{\mathrm{a}}$ \\
\hline Diferencias sobre la Pavana c. 98 & Sí & Corchea & $\mathrm{La}$ & $8^{\mathrm{a}}$ \\
\hline Fantasia XX c. 36 & No & Semínima & $\mathrm{La}$ & $5^{\mathrm{a}}$ \\
\hline Fantasía XXI cc. 7-8 & No & Semínima & Sol & $5^{\mathrm{a}}$ \\
\hline Fantasía XXII c. 184 & No & Semínima & $\operatorname{Re}$ & $6^{\mathrm{a}}$ \\
\hline Fantasía XXIII c. 41 & No & Corchea & $\mathrm{La}$ & $8^{\mathrm{a}}$ \\
\hline Fantasía XXIII c. 44 & No & Corchea & $\mathrm{La}$ & $4^{\mathrm{a}}$ \\
\hline Fantasía XXVIII c. 90 & No & Semínima & $\mathrm{La}$ & $3^{\mathrm{a}}$ \\
\hline Fantasía XXIX c. 92 & No & Corchea & $\mathrm{La}$ & $6^{\mathrm{a}}$ \\
\hline Fantasía XXIX c. 97 & No & Corchea & $\mathrm{La}$ & $6^{\mathrm{a}}$ \\
\hline Fantasía XXXIII c. 37 & No & \begin{tabular}{|l|} 
Corchea \\
\end{tabular} & $\mathrm{La}$ & $8^{\mathrm{a}}$ \\
\hline Soneto VI c. 36 & No & Semínima & $\operatorname{Re}$ & $5^{\mathrm{a}}$ \\
\hline Canción IV c. 64 & No & Semínima & Sol & $5^{\mathrm{a}}$ \\
\hline Soneto VII c. 35 & $\mathrm{No}$ & Semínima & $\operatorname{Re}$ & $3^{\mathrm{a}}$ \\
\hline Soneto VII c. 110 & $\mathrm{No}$ & Semínima & $\operatorname{Re}$ & $3^{\mathrm{a}}$ \\
\hline Soneto VII c. 132 & No & Semínima & $\operatorname{Re}$ & $6^{\mathrm{a}}$ \\
\hline Soneto XII c. 20 & No & Corchea & Sol & $5^{\mathrm{a}}$ \\
\hline Soneto XIX c. 3 & No & Corchea & $\mathrm{La}$ & $6^{\mathrm{a}}$ \\
\hline Soneto XIX c. 11 & No & Corchea & $\mathrm{La}$ & $6^{\mathrm{a}}$ \\
\hline Soneto XXIII c. 37 & No & Semínima & $\operatorname{Re}$ & Unísono \\
\hline Soneto XXVIII c. 37 & No & Semínima & $\operatorname{Re}$ & $5^{\mathrm{a}}$ \\
\hline Soneto XXVIII c. 44 & No & Semínima & $\operatorname{Re}$ & $5^{\mathrm{a}}$ \\
\hline Diferencias Vacas c. 116 & No & Semínima & $\mathrm{La}$ & $3^{\mathrm{a}}$ \\
\hline Diferencias Conde Claros & No & Corchea & $\operatorname{Re}$ & $6^{\mathrm{a}}$ \\
\hline
\end{tabular}

Así comprobamos que si bien la mayoría de veces se hace el movimiento de semitono, no faltan ejemplos de lo contrario. Pero prácticamente siempre que la nota donde se produce la bordadura forma un intervalo de $8^{\mathrm{a}}$ ó unísono, el movimiento de dicha bordadura es de semitono, como se comprueba en el ejemplo siguiente en los casos del primer sistema. Por el contrario, cuando el movimiento es de otro intervalo $\left(5^{\mathrm{a}}, 6^{\mathrm{a}}, 3^{\mathrm{a}}, 4^{\mathrm{a}}\right)$ no se altera la nota inferior y el movimiento es de tono, como en los casos del segundo sistema del ejemplo siguiente. Esto ocurre tanto cuando la nota es un $\mathrm{Re}$, un Sol o un $\mathrm{La}$ (o lo que es lo mismo, I, IV o V grados del primer tono, por ejemplo). 

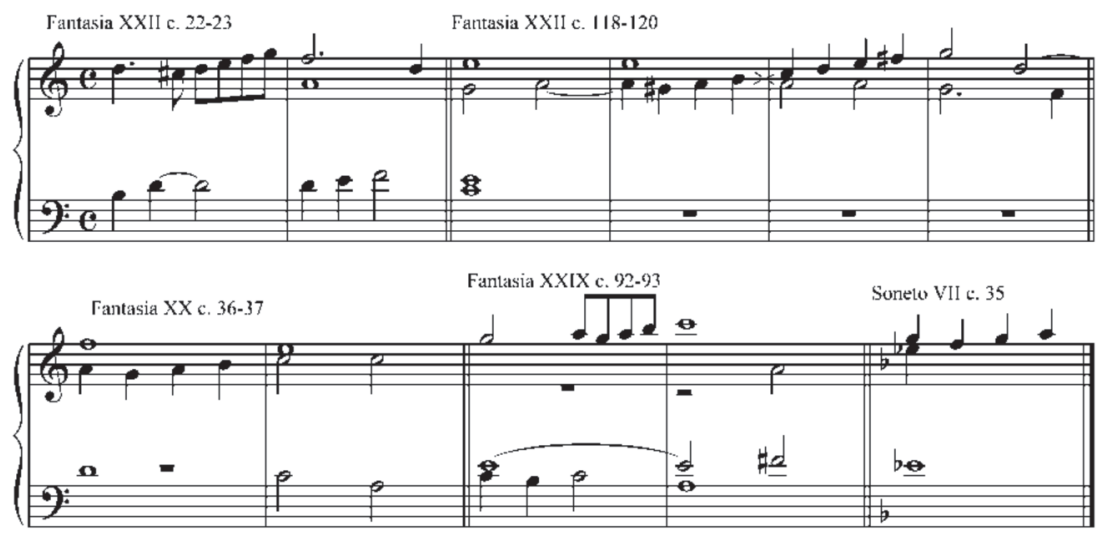

Ilustración 20: ejemplos de pasos de vuelta en Valderrábano ${ }^{34}$

Por dicha razón, se mantiene la sugerencia de \# para las notas marcadas con * en los ejemplos de Cabezón que se exponían al principio de este apartado (IluSe han marcado con* las bordaduras donde parece lógico que el movimiento sea de semitono. Siguiendo la propuesta metodológica de este trabajo, se ha examinado todas las bordaduras en re/ sol/la en contenidas en un grupo de obras de Valderrábano, excluyendo las que darían lugar a octava falsa. La tabla siguiente indica cuándo ese movimiento es de semitono (para lo cual se emplea el sostenido), en qué valores se mueve la glosa, a qué voz del hexacordo corresponde, y el intervalo que forma con otras voces, principalmente con el bajo. y otros similares.

\section{COHERENCIA EN GLOSA}

El problema que se toma ahora en consideración es cuando, debido a la glosa, se repite una nota de manera no contigua, y en una ocasión está alterada. Parece lógico que esté siempre alterada, para evitar la incoherencia de yuxtaponerse las formas natural o alterada de dicha nota. Los ejemplos siguientes corresponden a dichas situaciones, y las sugerencias de semitonía que se han hecho van en el sentido indicado.

34 VALDERRÁBANO, 1965, II: 9, 10, 1, 28, 44. 

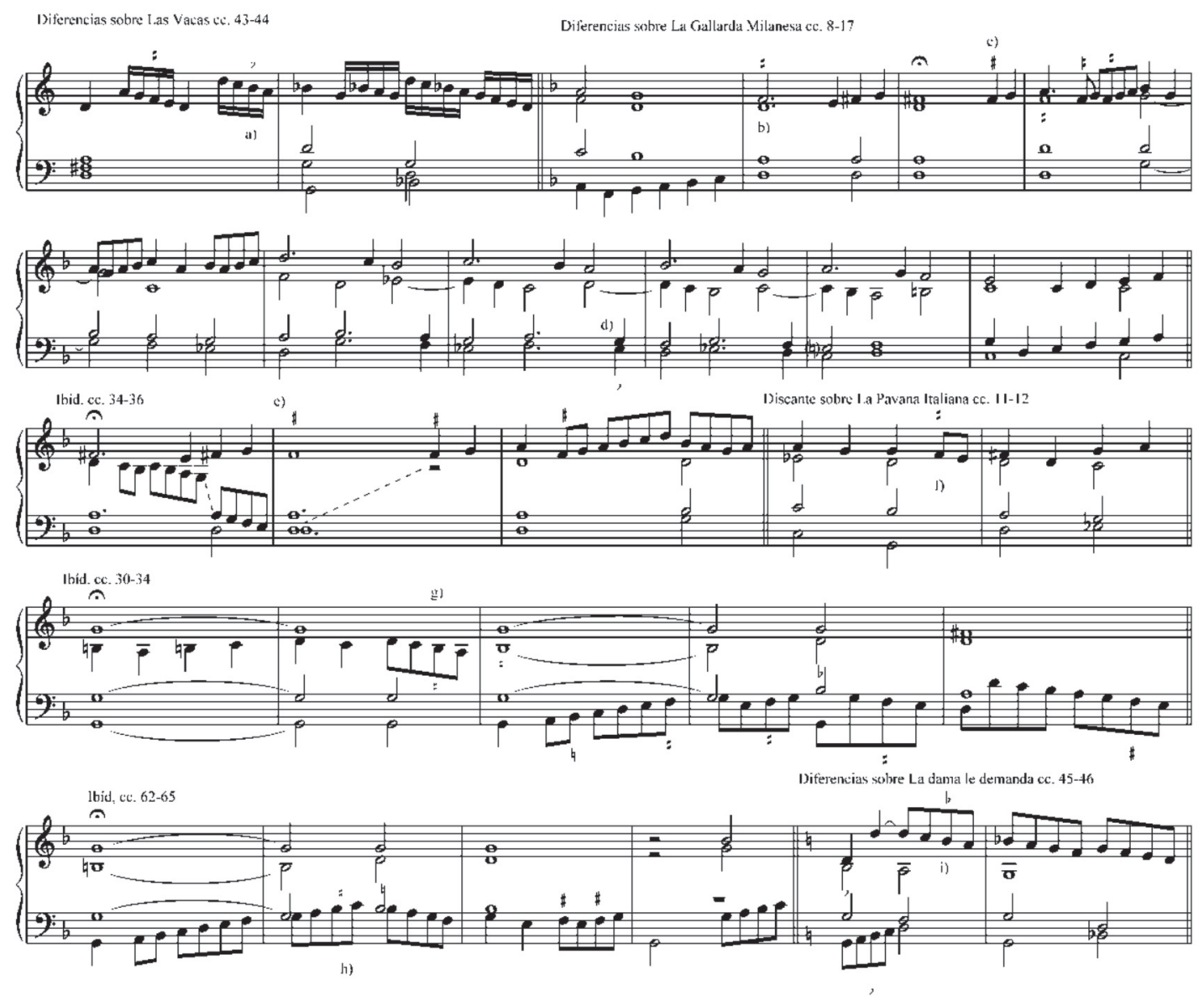

Ilustración 21: ejemplos de coherencia Cabezón ${ }^{35}$

Sin embargo, cabría plantearse que en la ejecución habría que respetar exactamente lo que dice la notación, por un prurito de fidelidad al "original", de no excedernos en nuestro celo de interpretar las antiguas fuentes. Pero antes habría que revisar el concepto de "lo que dice la notación" y de "original", aceptando como un principio que ni la notación trasmite de forma precisa todos los detalles, ni es su función hacerlo, ni existe el "original" de una música que tiene un amplio componente cinético.

Antes de examinar las fuentes vihuelísticas, conviene recordar que Diego Ortiz nos dice que una alteración tiene valor para toda una cláusula, no sólo para la nota concreta en la que está ubicada:

35 CABEZÓN, 2010, IV: 40-44, 50-53, 58-61, 62-65.
"Ha se de aduertir que quando en la clausula llana huuiere señalado sostenido que es este señal \# como en esta primera. que todos los puntos que en el contrapunto pasaren por alli sean sostenidos..." 36

Dicha afirmación se podría aplicar desde luego al caso f) de los anteriores (bordadura de una cláusula), pero también extrapolar a todos los demás.

Del examen de la literatura vihuelística se desprende que lo habitual es esta coherencia en la glosa. Son innumerables los casos en los que encontramos cláusulas en las que siempre las notas tienen la misma alteración, no encontrándose casos de lo contrario. Los siguientes solo son unos pocos ejemplos. 

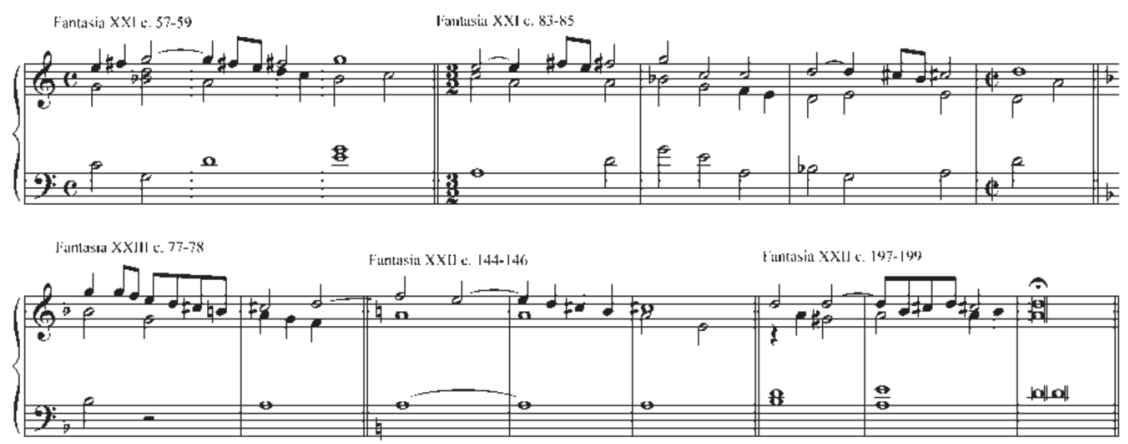

Ilustración 22: Ejemplos de Valderrábano que muestran la coherencia en las alteraciones en la glosa ${ }^{37}$

Se advierte en cualquier caso que los anteriores no son sino casos muy sencillos, en otros casos los cambios de dirección (ascendente-descendente) pueden justificar diferente alteración de las notas:

Ramillete, cuatro diferencias de folias cc. 17-19

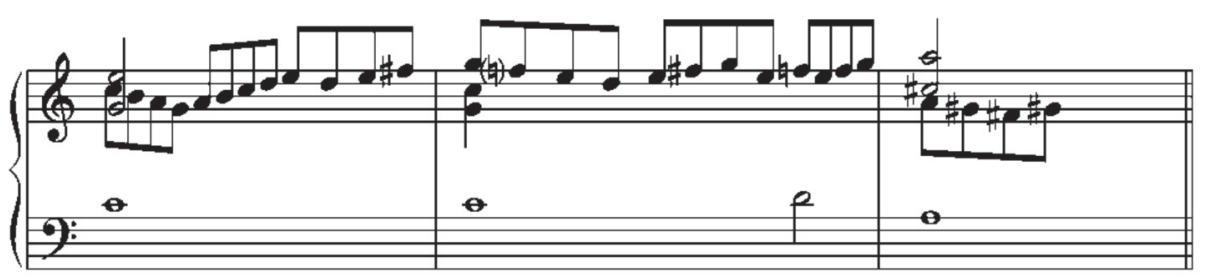

Ilustración 23: Cambio en las alteraciones debido al cambio de dirección ${ }^{38}$

\section{EL TRITONO EN LA GLOSA}

Obsérvese en el ejemplo anterior el tritono que crea el fa \# (Fefaut sustenido) al ascender, y cómo desaparece al descender. Con esto enlazo con la última cuestión que voy a tratar, a saber el caso de cuando la glosa pasa por una nota que forma un tritono con el bajo, es decir, que se emplee en la glosa el si natural (Befabemi sustenido B\#) sobre un fa (Fefaut, F), o el mi natural (Elami) sobre el si bemol (Befabemi bemol $\mathrm{Bb}$ ). Véanse los ejemplos siguientes.

37 VALDERRÁBANO, 1965, II: 3-5, 36, 7-11.

38 Ramillete, 1982: 87. 

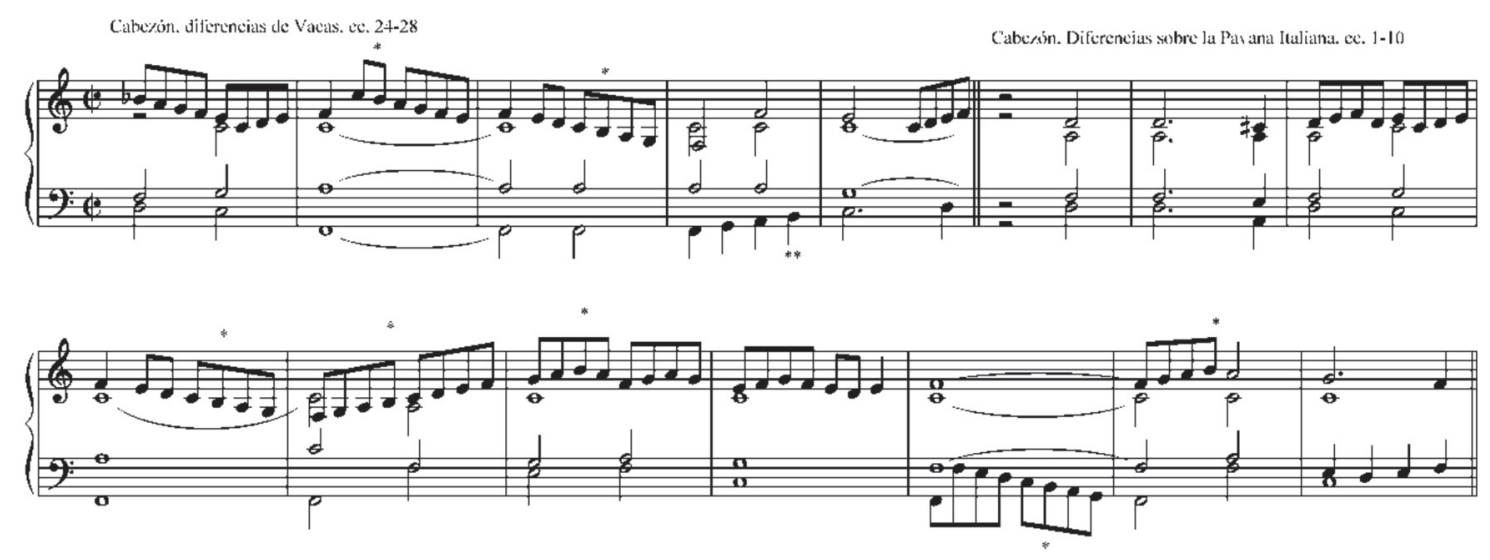

Caberón. Diferencias sobre l.a dama le demanda ce. 1-10

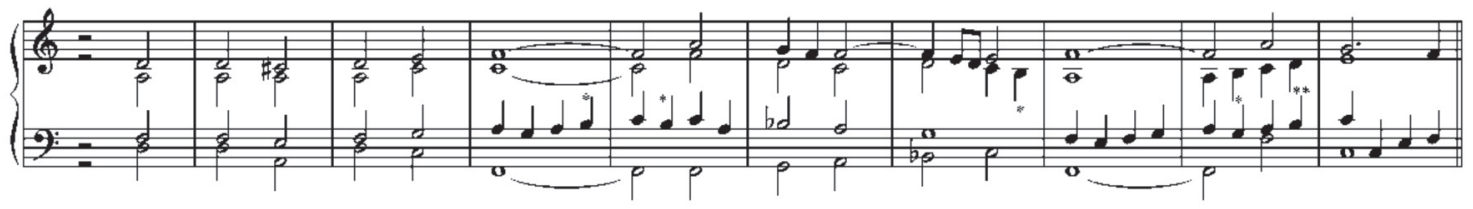

Ilustración 24: ejemplos de tritono en glosa en Cabezón ${ }^{39}$

El asterisco * señala lugares donde aparece un si natural (Befabemi), el cual parece lógico alterar con un bemol para evitar el tritono. No obstante, existe la duda de si se debe dejar natural, al menos los casos ascendentes. En ** habría si cabe todavía más duda de hacer bemol, al tratarse de un caso algo diferente, pues además de ascendente después cambia la consonancia.

En este caso, el examen de la literatura vihuelística nos arroja el resultado de que en general se tiende a evitar el tritono, especialmente al descender, alterando descendente- mente la nota correspondiente, como en los lugares marcados * del ejemplo siguiente. Sin embargo, sí se emplea abundantemente el tritono (es decir, no se emplea bemol) cuando la melodía asciende para ir a otra consonancia, como en los lugares marcados **. Se encuentran tritonos descendentes aunque muy ocasionalmente, como los mostrados en $* * *$. Estos se justifican por respetar coherencia en glosa, suelen ocurrir cuando sobre el IV grado en sexto tono, la voz superior glosa en torno al VII. 

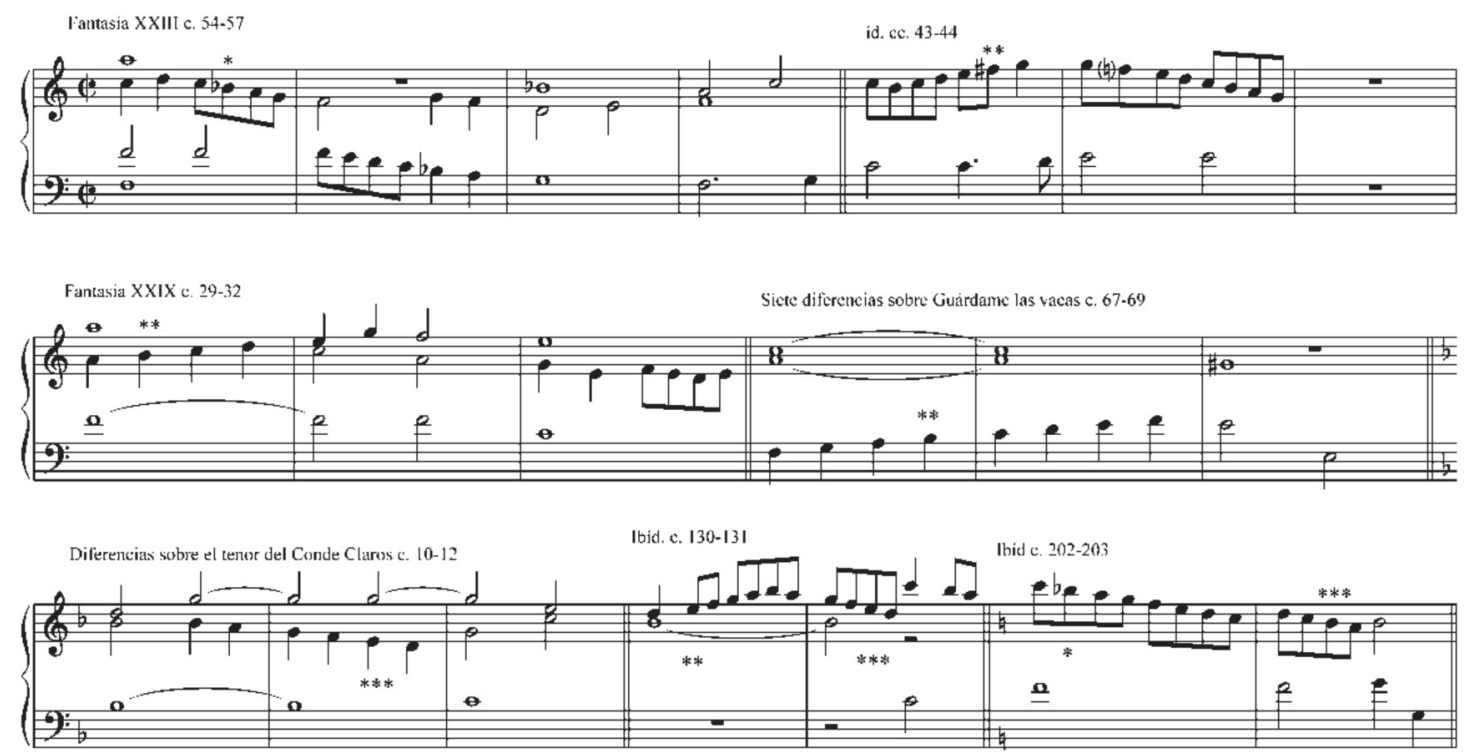

Ilustración 25: Tritonos evitados (*) o mantenidos (** y ***) en obras de Valderrábano ${ }^{40}$

Por estas razones, en general en la edición de Cabezón que realicé juntamente con Gerhard Doderer se optó por sugerir evitar el tritono en la glosa, aunque se respeta en movimientos ascendentes, como los marcados * en el ejemplo siguiente.

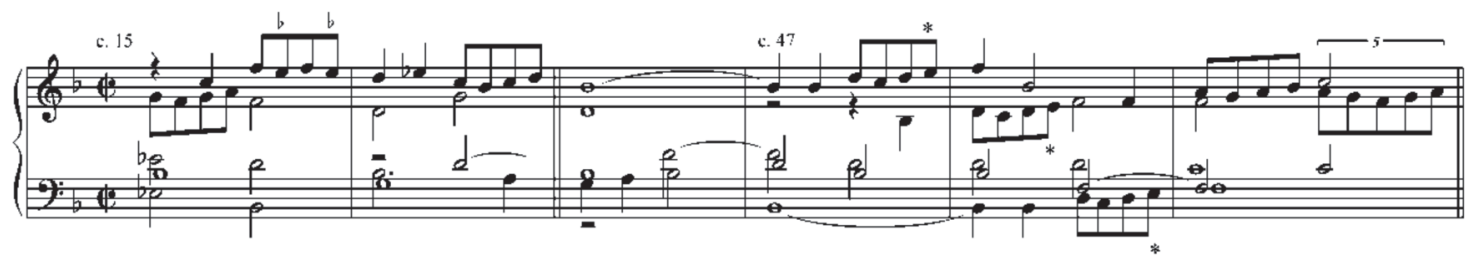

Ilustración 26: Tritonos en Susana de Hernando de Cabezón ${ }^{41}$

Aún así debo reconocer la dificultad de esta cuestión, y quizás admitir que podrían replantearse muchas de las sugerencias emitidas en la edición referida, especialmente en la música más eclesiástica, en la que el uso del tritono no siempre debe ser suavizado. Me remito a lo dicho por Correa y Sancta María (vide sopra, notas 8 y 12).

40 VALDERRÁBANO, 1965, II: 13, 27, 73, 75, 77, 86.

41 CABEZÓN, 2010, IV: 23-38.

\section{CONCLUSIONES}

La literatura vihuelística española del siglo XVI ofrece un razonable número de ejemplos en los que basar las decisiones para dirimir problemas de semitonía en la música de tecla de la misma época. La dificultad del tema y la enorme casuística no permiten elaborar un repertorio cerrado de soluciones, pero sí plantear este procedimiento como propuesta metodológica, que en cualquier caso hay que adoptar con prudencia. 
Aún así, del examen de un discreto número de casos concretos, se pueden establecer unos resultados, aunque sean provisionales:

- El $6^{\circ}$ grado en primer tono en una escala descendente después de la nota final debe mantenerse sin descender (como por el contrario parece pedirnos nuestro oído "tonal"). Sí debe alterarse descendentemente en escalas descendentes en el curso de la pieza.

- Tampoco el $6^{\circ}$ grado en el tono $1^{\circ}$ en la construcción IV-V-I debe ser siempre alterado descendentemente. Es cierto que es lo más habitual, pero también se encuentran casos de lo contrario.

- Al ascender por grados a una cláusula sostenida, la nota anterior a la cláusula debe estar ya sostenida.

- Las bordaduras o "pasos de vuelta" deben ser en general de semitono, cuando están en $8^{\mathrm{a}}$ con el bajo, y más usualmente de tono si forman otro intervalo $\left(3^{\mathrm{a}}, 5^{\mathrm{a}}, 6^{\mathrm{a}}\right)$.

- Se debe mantener la coherencia en la glosa, es decir, que una nota alterada en un fragmento esté siempre alterada, evitando que aparezca unas veces alterada y otras sin alterar. Excepción a esto es cuando hay un cambio de dirección ascendente-descendente: es corriente alterar ascendentemente al subir y eliminar la alteración al descender.

- Se debe evitar el tritono en la glosa, excepto cuando se asciende a una consonancia diferente.

Es posible que estos resultados puedan parecer naturales y lógicos, y quizás el lector piense que se trata de propuestas ya conocidas. En realidad la finalidad de este artículo no es tanto dar a conocer esas pautas, que seguro manejan ya de forma intuitiva los organistas y clavecinistas por su experiencia con el repertorio. Se trata de fundamentarlas en el hecho de que se comprueba que las soluciones adoptadas se corresponden con la práctica de la época en la literatura vihuelística, donde razonablemente podemos suponer que tenemos una versión con las alturas correctas debido a la naturaleza de su tablatura.

Debe necesariamente concluirse reconociendo una vez más que la cuestión está abierta, y que los resultados no deben ser tomados como una receta simplista.

\section{BIBLIOGRAFÍA}

APEL, Willy. Accidentien und Tonalität in den Musikdenkmälern des 15. Und 16. Jahrhunderts, Berlin 1936.

ARLETTAZ, Vincent, Musica Ficta. Une histoire des sensible du XIIIe au XVIs siècles, Sprimont (Bélgica), Ed. Mardaga, 2000.
BERMUDO, Juan, Declaración de Instrumentos Musicales, Osuna, Juan de León, 1555.

BERNAL, Miguel, "Francisco Correa de Arauxo, teórico de la seconda prattica: Tratamiento de la disonancia y casuística moral", Revista de Musicología, XXVIII, 2 (2005): 891-917.

CABEZÓN, Hernando, Obras de Música para Tecla, Arpa y Vihuela, Madrid, Francisco Sánchez, 1578. Ed. moderna Miguel Bernal Ripoll y Gerhard Doderer, Kassel, Bärenreiter, 2010.

CERONE, Pietro, El Melopeo y Maestro, Nápoles, J. B. Gargano y L. Nucci, 1613.

DAZA, Esteban, Libro de música en cifras para Vihuela, intitulado el Parnasso, Valladolid: Diego Fernández de Córdoba, 1576. Ed. Moderna: Los Vihuelistas. Esteban Daça. Rodrigo de Zayas, Madrid, Alpuerto, 1983.

HAKALAHTI, Iina-Karita, Maestro Francisco Correa de Arauxo's (1584-1654) Facultad Orgánica (1626) as a source of performance practice, Helsinki, Helsinki University Print, 2008.

JACOBS, Charles, "Spanish Renaissance Discussion of Musica Ficta", Proceedings of the American Philosophical Society, 112 (Philadelphia, 1968): 277-298.

JACOBS, Charles, La interpretación de la música española del siglo XVI para instrumentos de teclado, Madrid, Dirección general de relaciones culturales, 1959.

KASTNER, Macario Santiago, "Semitonia-Probleme in der iberischen Claviermusik des 16. und 17. Jahrhunderts", Anuario Musical, 47 (Barcelona, 1968): 3-33. Traducción al castellano en Joan Baptista Cabanilles Músico valenciano universal, Valencia: ACAO, 1981: 119-152.

LAMBEA, Mariano, "La cuestión de la semitonía subintelecta en Cerone (El melopeo y maestro, 1613)", Nassarre, 12-2 (Zaragoza, 1996): 197-216.

LORENTE, Andrés, El porqué de la Música, Alcalá de Henares, Nicolás de Xamares, 1672. Ed. facsímil José Vicente González Valle, Barcelona, CSIC, 2002.

MILÁN, Luys, Libro de Música de Vihuela de Mano. Intitulado El Maestro. Valencia: Francisco Díaz Roman, 1536. Ed. facsímil Francisco Roa. Madrid: Sociedad de la Vihuela, 2008. Ed. Moderna Ruggero Chiesa. Milán: Suvini Zerboni, 1974.

MUDARRA, Alonso. Tres libros de música en cifra para vihuela. Sevilla: Juan de León, 1546. Edición moderna, Emilio Pujol, Barcelona, CSIC, 1984.

NASARRE, Pablo. Escuela Música según la práctica moderna Vol.I, Zaragoza, Herederos de Diego de Larumbe, 1724. Ed. Facsímil L. Siemens, Zaragoza, Institución Fernando el Católico, 1980. 
NASARRE, Pablo. Fragmentos Músicos. Madrid, Imprenta Real, 1700 ( $1^{\text {a }}$ edición Zaragoza, 1683). Ed. Facsímil A. Zaldívar, Zaragoza, Institución Fernando el Católico, 1988.

ORTIZ, Diego. Trattado de Glosas sobre Cláusulas y otros géneros de puntos en la Música de Violones. Roma, Valerio \& Luis Dorico, 1553. Ed. facsímil Max Schneider, Kassel/Basel/Tours/London, Bärenreiter, 1936.

PISADOR, Diego, Libro de Música de Vihuela, Salamanca: el autor, 1552. Ed. Facsímil y transcripción moderna F. Roa/F. Gértrudix. Madrid: Pygmalión, 2002.

Ramillete de flores (1593), E:Mn Ms 6001. Edición moderna Ramillete de flores, Juan José Rey, Madrid: Alpuerto, 1982.

RUBIO, Samuel, La polifonía clásica, El Escorial, La ciudad de Dios, 1956.

SANCTA MARIA, Tomás de. Arte de Tañer fantasía. Valladolid, Francisco Fernández de Córdoba, 1565.
SILBIGER, Alexander, "Introduction: The first centuries of European Keyboard Music", Alexander Silbiger ed., Keyboard music before 1700, New York/London, Routledge, 2004 ( $1^{\text {a }}$ edición 1995).

TOVAR, Francisco. Libro de música práctica. Barcelona, Johan Rosenbach, 1510.

VALDERRÁBANO, Enríquez de, Libro de música de vihuela intitulado Silva de Sirenas, Valladolid, Francisco Fernández de Córdoba, 1547. Edición Moderna Emilio Pujol, Barcelona, CSIC, 1965.

VENEGAS DE HENESTROSA, Luis, Libro de cifra nueva para tecla, harpa y vihuela, Alcalá, Joan de Brocar, 1557.

WYLY, James, The Pre-Romantic Spanish Organ: Its Structure, Literature, an Use in Performance, D.M.A. dissertation, University of Missouri at Kansas City, 1964.

Recibido: 29.10 .2012

Aceptado: 29.10.2012 